\title{
Directed Energy Deflection Laboratory Measurements
}

\author{
Travis Brashears $^{(1)}$, Philip Lubin ${ }^{(1))}$, Gary B. Hughes ${ }^{(2)}$, Peter Meinhold ${ }^{(1)}$, Jonathan Suen ${ }^{(1)}$, \\ Payton Batliner $^{(1)}$, Caio Motta ${ }^{(1)}$, Janelle Griswold ${ }^{(1)}$, Miikka Kangas ${ }^{(1)}$, Isabella \\ Johansson $^{(1)}$, Yusuf Alnawakhtha ${ }^{(1)}$, Kenyon Prater ${ }^{(1)}$ Alex Lang ${ }^{(1)}$, and Jonathan \\ Madajian $^{(1)}$ \\ (1)Physics Department, University of Santa Barbara, CA 93106, trbrashears@gmail.com, +1- \\ 805-893-8432 \\ ${ }^{(2)}$ Statistics Department, California Polytechnic State University, San Luis Obispo, CA 93407, \\ gbhughes@calpoly.edu, +1-805-756-5648
}

KEYWORDS: Planetary defense, directed energy, orbital deflection via laser, laboratory thrust measurements

\begin{abstract}
We report on laboratory studies of the effectiveness of directed energy planetary defense as a part of the DE-STAR (Directed Energy System for Targeting of Asteroids and exploRation) program. DE-STAR [1][5][6] and DE-STARLITE [2][5][6] are directed energy "stand-off" and "stand-on" programs, respectively. These systems consist of a modular array of kilowatt-class lasers powered by photovoltaics, and are capable of heating a spot on the surface of an asteroid to the point of vaporization. Mass ejection, as a plume of evaporated material, creates a reactionary thrust capable of diverting the asteroid's orbit. In a series of papers, we have developed a theoretical basis and described numerical simulations for determining the thrust produced by material evaporating from the surface of an asteroid [1][2][3][4][5][6]. In the DE-STAR concept, the asteroid itself is used as the deflection "propellant". This study presents results of experiments designed to measure the thrust created by evaporation from a laser directed energy spot. We constructed a vacuum chamber to simulate space conditions, and installed a torsion balance that holds an "asteroid" sample. The sample is illuminated with a fiber array laser with flux levels up to $60 \mathrm{MW} / \mathrm{m}^{2}$ which allows us to simulate a mission level flux but on a small scale. We use a separate laser as well as a position sensitive centroid detector to readout the angular motion of the torsion balance and can thus determine the thrust. We compare the measured thrust to the models. Our theoretical models indicate a coupling coefficient well in excess of $100 \mu \mathrm{N} / \mathrm{W}_{\text {optical, }}$, though we assume a more conservative value of 80 $\mu \mathrm{N} / \mathrm{W}_{\text {optical }}$ and then degrade this with an optical "encircled energy" efficiency of 0.75 to $60 \mu \mathrm{N} / \mathrm{W}_{\text {optical }}$ in our deflection modeling. Our measurements discussed here yield about $45 \mu \mathrm{N} / \mathrm{W}_{\text {absorbed }}$ as a reasonable lower limit to the thrust per optical watt absorbed.
\end{abstract}

TABLE OF CONTENTS

1. INTRODUCTION .......................................................2

2. ASTEROID IMPACT THREAT ..............................2

3. 3D THERMAL ANALYSIS AND 2D MODELS ......2

3.1 MODELING RESULTS .............................4

4. EXPERIMENTAL DESIGN........................................5

5. LASER POWER ......................................................9

6. MEASUREMENTS RESULTS ............................10

6.1 Mass EjeCtion PHOTOS \& Video.12

7. CONCLUSIONS AND FUTURE WORKS ..............12 


\section{INTRODUCTION}

This paper gives the results from laboratory tests of directed energy laser ablation for mitigation of asteroids and comets. We present both the theory of and measurements of laser driven ablation. We first present the theoretical models of 2D analytic and 3D numerical simulations, then the laboratory measurements and compare the results. Once the foundation of the theoreticalexperimental comparison is laid out the experimental setup to measure laser ablation thrust is outlined in detail. The motivation behind implementing a directed energy planetary defense system is discussed in detail in our other papers related to the DE-STAR program [1][2][3][4][5][6].

\section{ASTEROID IMPACT THREAT}

Asteroid impacts pose a continual threat to modern civilization. On 15 February 2013, an asteroid penetrated the atmosphere over Chelyabinsk, Russia entering at an angle of approximately $18^{\circ}$, and releasing energy equivalent to $570 \pm 150 \mathrm{kt}$ TNT [7]. For comparison, the nuclear weapon that was detonated approximately $509 \mathrm{~m}$ above the ground in Hiroshima, Japan yielded approximately $12.5 \mathrm{kt} \mathrm{TNT} \mathrm{[8].} \mathrm{The} \mathrm{main} \mathrm{airburst} \mathrm{over} \mathrm{Chelyabinsk} \mathrm{occurred} \mathrm{at} \mathrm{an} \mathrm{approximate} \mathrm{altitude} \mathrm{of} 30 \mathrm{~km}$ and created a shock wave strong enough to shatter windows out to a distance of $120 \mathrm{~km}$ from the meteorite's track, injuring over 1,200 people in Chelyabinsk city and hundreds more in nearby towns and rural areas [7]. Had the asteroid approached from a higher angle, more serious damage would be anticipated from higher concentration of the impact energy on the ground.

Sixteen hours after the meteorite struck near Chelyabinsk, the 45 m diameter asteroid 2012 DA14 approached to within $27,743 \mathrm{~km}$ of Earth's surface - inside the orbit of geosynchronous satellites. If DA14 were to strike Earth, it would deliver approximately 7.2 Mt TNT [9]. Although the Chelyabinsk meteorite and DA14 arrived at or near Earth on the same day, the two objects were not linked to each other, coming from completely unrelated orbits. The fact that two such seemingly improbable events could occur within hours of each other serves as a stark reminder that humanity is continually at risk of asteroid impact.

Asteroids at least the size of DA14 ( $\sim 0 \mathrm{~m}$ diam.) are expected to strike Earth approximately every 650 years, while objects at least the size of the Chelyabinsk impactor ( $20 \mathrm{~m} \mathrm{diam}$.) are expected to strike Earth approximately every 100 years [9]. Larger objects also pose a severe threat, as the total kinetic energy associated with an impact of a $100 \mathrm{~m}$ asteroid is equivalent to approximately $85 \mathrm{Mt} \mathrm{TNT}$, and that of the well-known $325 \mathrm{~m}$ threat, Apophis, is approximately 3.2 Gt TNT [9]. Thus, effective mitigation strategies are imperative to ensure humanity's continuity and future advancement.

\section{3D THERMAL ANALYSIS AND 2D MODELS}

We calculate the thrust produced by directed energy focused on an asteroid using two different modeling approaches (2D analytic and 4D (3D + time) numerical), of increasing complexity and realism. The basic equations are derived from energy conservation and mass flow and are covered in detail in our other papers [1][5][6]:

Power in $($ laser $)=$ Power out $($ radiation + mass ejection $)+\frac{d U}{d t}$

Where $U=$ Asteroid internal energy and $\frac{d U}{d t}$ is effectively from conduction.

In the steady state $\frac{\mathrm{dU}}{\mathrm{dt}}=0$

$P_{\text {in }}=P_{\text {out }}+\frac{d U}{d t}$, with $U=\int \rho c_{v} d v$

Where $\mathrm{c}_{\mathrm{v}}=$ specific heat $[\mathrm{J} / \mathrm{kg}-\mathrm{K}]$, 
$\mathrm{F}_{\mathrm{L}}=$ Laser flux $\left[\mathrm{W} / \mathrm{m}^{2}\right]-($ in $), \mathrm{F}_{\text {cond }}=$ Thermal conduction $\left[\mathrm{W} / \mathrm{m}^{2}\right]-($ in $)$, and

$\mathrm{F}_{\text {rad }}=$ Radiation flux $\left[\mathrm{W} / \mathrm{m}^{2}\right]-($ out $), \mathrm{F}_{\text {ejecta }}=$ Ejecta flux $\left[\mathrm{W} / \mathrm{m}^{2}\right]-($ out $)$.

Assuming $\mathrm{P}_{\text {in }}=\mathrm{P}_{\text {rad }}+\mathrm{P}_{\text {Ejecta }}+\mathrm{P}_{\text {cond }}$, then:

f] $\left(\mathrm{F}_{\mathrm{L}}-\overline{\mathrm{F}}_{\text {rad }}-\overline{\mathrm{F}}_{\text {Ejecta }}-\overline{\mathrm{F}}_{\text {cond }}\right) \times \hat{n} \mathrm{dA}=0$

or $=\int \tilde{N} \times\left(\bar{F}_{L}-\bar{F}_{\text {rad }}-\bar{F}_{\text {Ejecta }}-\bar{F}_{\text {cond }}\right) \mathrm{dV}=0$

Locally:

$\overrightarrow{\mathrm{F}}_{\mathrm{L}}=\overrightarrow{\mathrm{F}}_{\text {rad }}+\overrightarrow{\mathrm{F}}_{\text {Ejecta }}+\overrightarrow{\mathrm{F}}_{\text {cond }}$

$\overrightarrow{\mathrm{F}}_{\mathrm{rad}}=\sigma \mathrm{T}^{4} \hat{\mathrm{n}}$

$\overline{\mathrm{F}}_{\mathrm{Ejecta}}=\Gamma \mathrm{e} \mathrm{H}_{\mathrm{v}} \hat{n}=M^{1 / 2}(2 \pi R T)^{-1 / 2} \alpha_{\mathrm{e}} 10^{[A-B /(T+C)]} H_{v} \hat{n}$

$\left|\overline{\mathrm{F}}_{\text {cond }}\right|=\mathrm{KN} \mathrm{N} T$,

$\left|\bar{F}_{\text {rad }}\right|=\sigma T^{4}$, and $\left|\bar{F}_{\text {Ejecta }}\right|=\Gamma e \times H_{v}$.

Where $\mathrm{K}$ is the thermal conductivity (which can be position and temperature dependent) and $\Gamma e$ is the mass ejection flux $\left[\mathrm{kg} / \mathrm{m}^{2}-\mathrm{s}\right]$, and $\mathrm{H}_{\mathrm{v}}$ is the heat of vaporization $[\mathrm{J} / \mathrm{kg}]$. The heat of fusion, $\mathrm{H}_{\mathrm{f}}$, is included for relevant cases. The heat of fusion is sometimes referred to the heat of sublimation as is sometimes the case for compounds in vacuum. $\mathrm{H}_{\mathrm{f}}$ is typically a small fraction of $\mathrm{H}_{\mathrm{v}}$. The mass ejection flux is shown in equation 16 which uses vapor pressure.

$\Gamma e=\frac{M \alpha_{e}\left(P_{v}-P_{h}\right)}{\sqrt{2 \pi M R T}}=M^{1 / 2}(2 \pi R T)^{-1 / 2} \alpha_{e}\left(P_{v}-P_{h}\right)$

$\mathrm{M}=$ Molar mass $[\mathrm{kg} / \mathrm{mol}]$

$\mathrm{P}_{\mathrm{v}}=$ Vapor pressure $[\mathrm{Pa}]$

$\mathrm{P}_{\mathrm{h}}=$ Ambient vapor pressure $=0$ (in vacuum)

$\alpha_{e}=$ coef. of evaporation

The models vapor pressure for each element and compound is determined using a semi analytic form known as Antoine coefficients $\mathrm{A}, \mathrm{B}$ and $\mathrm{C}$ in equation 17.

$\operatorname{LOG}\left(P_{\mathrm{v}}\right)=\mathrm{A}-\mathrm{B} /(\mathrm{T}+\mathrm{C})$

Where $\mathrm{A}, \mathrm{B}$ and $\mathrm{C}$ are unique per element and compound. Hence:

$P_{v}=10^{[A-B /(T+C)]}$ and $\left.\left|F_{E j e c t a}\right|=M^{1 / 2} \frac{1}{\sqrt{2 \pi R T}} \alpha_{e} 10^{[A-B /(T+C)}\right] H_{v}$

A Gaussian profile is assumed for the laser as an approximation shown in equation 19 where the Gaussian laser power is $\mathrm{P}_{\mathrm{T}}$, and $\mathrm{r}$ is the distance from the spot center.

$\left|\bar{F}_{\mathrm{L}}\right|=\frac{\mathrm{P}_{\mathrm{T}}}{2 \pi \sigma^{2}} \mathrm{e}^{-\mathrm{r}^{2} / 2 \sigma^{2}}$

In the approximation where the spot is small compared to the asteroid, the equation becomes:

$\vec{F}_{L}=\frac{-P_{T}}{2 \pi \sigma^{2}} e^{-r^{2} / 2 \sigma^{2}} \hat{n}$ 
In the dynamic case it is possible to solve for transient heat flow by :

$\tilde{N} \times(K \tilde{N} T)+\frac{d}{d T}\left(\rho c_{v} T\right)=0$

$K \tilde{N}^{2} T+\rho c_{v} \frac{d T}{d t}=0$

In equation 21 it is assumed that $\mathbf{K}$ (thermal conductivity) is independent of position, $\rho$ and $\mathrm{c}_{\mathrm{v}}$ are time independent. In the 2D steady state solutions, the thermal conductivity is assumed to be small (this is shown to be a valid assumption from first principle calculations) and a combination of radiation and mass ejection (phase change) is used:

$\left|\overrightarrow{F_{L}}\right|=\left|\overrightarrow{F_{\text {rad }}}\right|+\left|\overrightarrow{F_{\text {Ejecta }}}\right|=F_{T}$

$\left.F_{T}=\sigma T^{4}+M^{1 / 2}(2 \pi R T)^{-1 / 2} 10^{[A-B /(T+C)}\right] H_{v}$

Inversion is not analytically tractable so numerical inversion is used to get $T\left(F_{T}\right)$ which gives $P_{V}\left(F_{T}\right), \Gamma e\left(F_{T}\right)$ etc. In this inversion, a function fit is found (to 10th order typically);

$T=\sum_{n=1}^{N} a_{n}\left(\log F_{T}\right)^{n}$

Gaussian approximation to the laser profile is used (this is not critical) to get $T(r), P_{v}(r), \Gamma_{e}(r)$ where $\mathrm{r}$ is the distance from the center of the spot.

Since radiation goes as the $4^{\text {th }}$ power of $\mathrm{T}$, while the mass ejection from evaporation goes roughly exponentially in $\mathrm{T}$, at low flux levels the outward flow is completely dominated by radiation (the asteroid is heated slightly and it radiates). As the spot flux level increases (spot size shrinks or power increases or both) evaporation becomes increasingly dominant and eventually at about $\mathrm{T}$ $2,000-3,000 \mathrm{~K}$ or fluxes of $10^{6}-10^{7} \mathrm{~W} / \mathrm{m}^{2}$ mass ejection by evaporation becomes the dominant outward power flow in rocky materials and (just as water boiling on a stove) the temperature stabilizes and increasing flux only increases the rate of mass ejection with only very small increases in temperature [1][5][6]. Fir comets, which contain lower temperature volatiles the temperatures and fluxes required to achieve mass ejection domination is much lower. Hence the results that follow are for the more difficult cases of high temperature materials.

\subsection{Modeling RESUlts}

We discuss the detailed thermal conductivity and rotation models in our papers [1][5][6]. Generally speaking small asteroids have larger thermal conductivity and are molecularly bound and thus can rotate faster before rotational breakup, while larger asteroid $(>100 \mathrm{~m}$ in diameter) have lower thermal conductivity and are gravitational bound and rotate more slowly. Using the equations above and the 2D numerical inversions and the full 3D and 4D simulations it is possible to solve for the temperature distribution and thus the mass ejection and thrust on the asteroid among many other parameters. We assume a Gaussian beam for simplicity. The parameter $\sigma$ (sigma) in the Gaussian beam profile is allowed to vary to show the effects of non-ideal beam formation as well as beam and pointing jitter. At higher powers the system is quite tolerant to errors in beam formation, focus, beam jitter and pointing errors [1][5][6]. The requirements on a low power system at equivalent distances are more severe. These relationships also show that it is possible to nearly achieve the theoretical maximum mass ejection rate. Our calculations and numerical simulations show the coupling to be between 100 and $500 \mu \mathrm{N} / \mathrm{W}_{\text {optical }}$ depending on the asteroid material composition and the laser flux and power on target we use [1]. This is comparable to the Shuttle SRB in thrust per watt which is closer to $1000 \mu \mathrm{N} / \mathrm{W}$ where the power here is the chemical engine power of the exhaust. This is not surprising, considering that conventional propellants are 
approximately thermal in nature with temperatures close to the maximum sustainable in the combustion chamber and exhaust nozzle (i.e., a few $\times 10^{3} \mathrm{~K}$ ) which is about the same temperature we achieve in the central spot for high temperature materials.

We assume more conservative numbers for system performance, typically $80 \mu \mathrm{N} / \mathrm{W}_{\text {optical. }}$ More laboratory measurements are needed for various materials and flux levels. For now we assume this

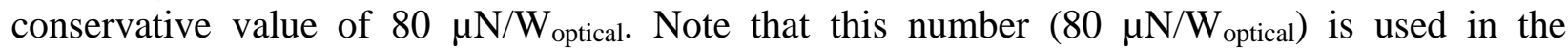
simulations of the asteroid deflection and orbital trajectories in our other papers. The $\mathrm{W}_{\text {optical }}$ is the laser power emitted. We generally make an additional assumption of an encircled energy fraction (fraction of emitted laser power in the central spot) of 0.75 thus yielding about $60 \mu \mathrm{N} / \mathrm{W}_{\text {optical }}$ or $60 \mu \mathrm{N}$ of thrust per optical watt emitted. In the simulations below we assume that this is all absorbed. Below, we show laboratory measurements that allow us to estimate a coupling of about $45 \mu \mathrm{N} / \mathrm{W}_{\text {absorbed }}$ where the optical power absorbed by the target $\left(\mathrm{W}_{\text {absorbed }}\right)$ includes estimated surface melt reflection and ejecta debris scattering. We have also run numerous 4D (3D spatial + time) numerical simulations that show good agreement between the 2D and 3D methods [4][5][6]. In all cases the coupling between laser power and thrust achieved will also be material dependent, though our simulations of many materials indicate we should achieve well above $80 \mu \mathrm{N} / \mathrm{W}_{\text {optical }}$ in the an optimized system for most materials expected in asteroids. These measurements and simulations are part of an ongoing effort in our group. As laboratory tests are refined, the results will then feed back into the models for various materials. Figure 1 shows some of the simulations for low power systems (1-1000 kw) typical of a DE-STARLITE mission.

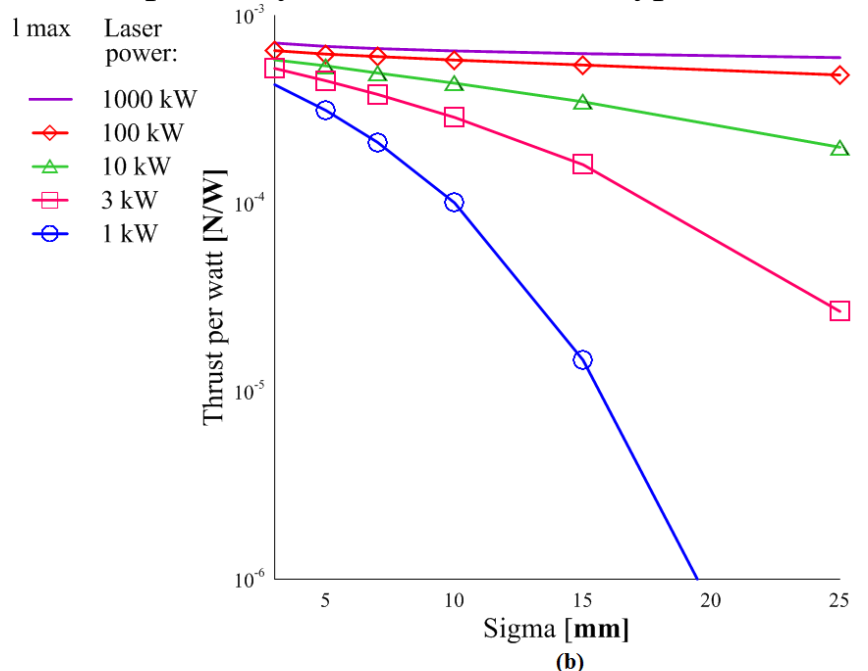

Figure 1. 2D analytic model results using $\mathrm{SiO} 2$ as the equivalent material. (a) Integrated mass ejection rates vs. sigma case for different powers between $1 \mathrm{~kW}$ and $1 \mathrm{MW}$. (b) Similarly, integrated thrust $(\mathrm{N})$ per watt vs. sigma.

\section{EXPERIMENTAL DESIGN}

The theoretical models outlined in the above section require testing which will enable better modeling. A fundamental step in moving forward with is then to compare our theoretical models with experimental results. We have constructed a number of laboratory experiments and data numerous data sets now in order to accomplish this. Analysis of laser power, ablation at varying pressure under vacuum, and thrust of ablation have all been tested multiple times and are reasonably consistent. Though much more remains to be done we summarize these results here. In order to measure the thrust from ablation a sensitive vacuum torsion balance system 
was designed a built in several generations of increasing sophistication. The system in shown in Figures 2 and 3.

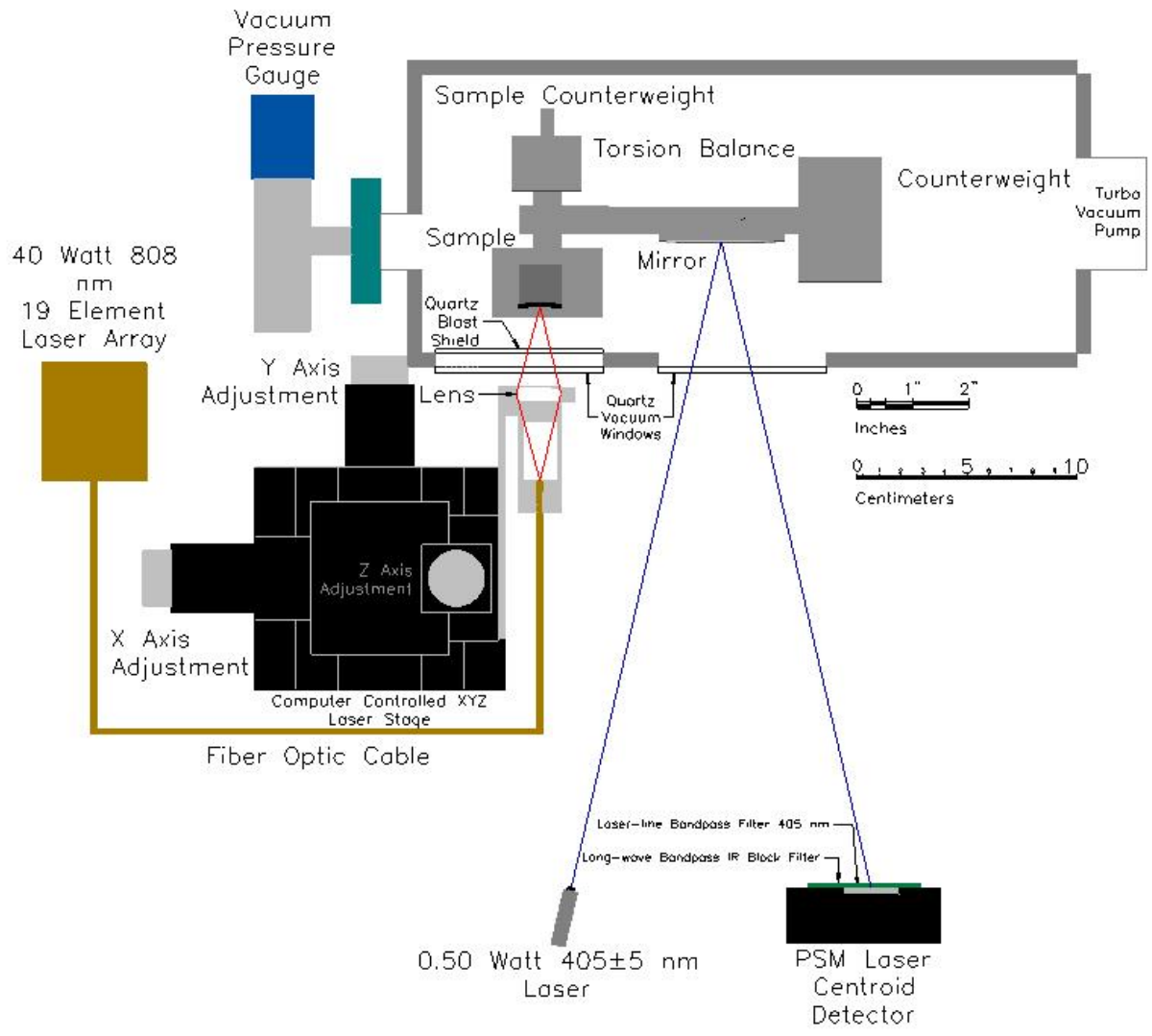

Figure 3. Top-view model of experimental setup. Ablation laser feeds through a fiber optic cable, through a lens, and past 2 quartz windows to ablate the sample on a torsion balance. As the torsion balance rotates the measurement laser reflects off a mirror and moves along the PSM.

\subsection{LASER}

A 19 element laser array with max power of 40 Watts, operating at $808 \mathrm{~nm}$ is mounted to a set of thermoelectric coolers that is then fed through an 810 micron diameter fiber optic cable attached to an anti-reflection coated lens that directs the laser beam onto the surface of the sample. As the ablation process takes place, the laser bores a hole into the sample. In order to keep the laser's focal point on the surface of the sample, an XYZ stage is implemented to allow hand or computer adjustments of the target spot. (Figure 3) 


\subsection{TORSION BALANCE}

The vacuum torsion balance system was machined to allow adjustable counterweights to adjust the sample balance in the horizontal and vertical axis. The torsion balance is attached to a stainless steel torsion fiber that is $1.64 \mathrm{~mm}$ in diameter. The fiber is enclosed in a vacuum tube that is fixed to an angular micrometer to center the readout beam on the readout detector. (Figure 2, 3, 4)

\subsection{ACQUISITION DESIGN}

The angular position of the torsion balance is measured using $405 \mathrm{~nm}$ adjustable focus laser that is directed at a mirror attached at the center of the torsion balance that reflects into a Silicon $2 \mathrm{D}$ position sensitive centroid detector (PSD). The detector has an area of $100 \mathrm{~mm}^{2}$ $(10 \times 10 \mathrm{~mm})$ and measures the position of the centroid

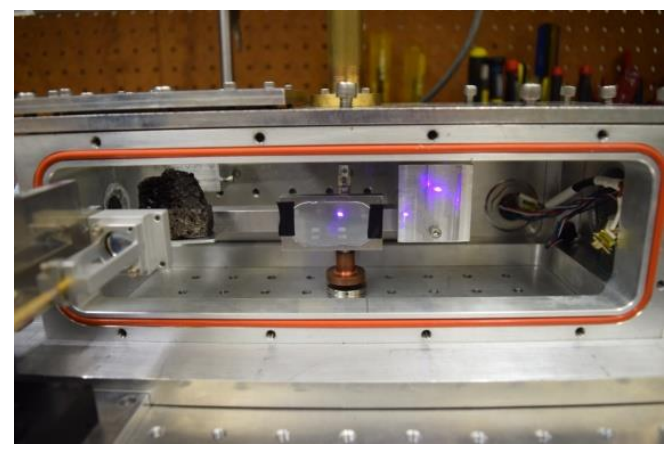

Figure 4. Torsion balance with sample, counterweight, and Ablation Laser aimed at sample. Measurement laser can be seen on mirror. Eddy current dampener shown at bottom of chamber. (optical spot center of gravity) of the readout laser spot to within a few microns. When a thrust is applied to the sample the readout laser moves along the PSD detector and outputs a voltage proportional to the displacement. While taking data of laser ablation, to avoid light leakage from the 40 Watt $808 \mathrm{~nm}$ into the PSD detector two shortwave pass filters were implemented: laser-line band pass filter and a long-wave band pass filter. These filters reduce the leakage by more than 10 orders of magnitude and are critical. The PSD detector output is amplified, conditioned (low pass) and connected to a data acquisition system that is sampled at $10 \mathrm{~Hz}$ with 16 bit ADC resolution. Further software filtering gives the final measurement results.

\subsection{VACUUM ChAMber AND PUMP}

The vacuum chamber was designed to simulate a space like environment and shield the IR laser from the ejected debris. The system is pumped by a turbomolecular pump. The high power ablation laser is external to the vacuum chamber and is focused externally on the target. The beam passes through an outer quartz vacuum window and then through an inner quartz blast shield. The inner blast shield prevents the high speed ejecta from damaging the outer vacuum window to prevent catastrophic failure. A second quartz window is located at the middle of the chamber for the torsion balance readout laser to

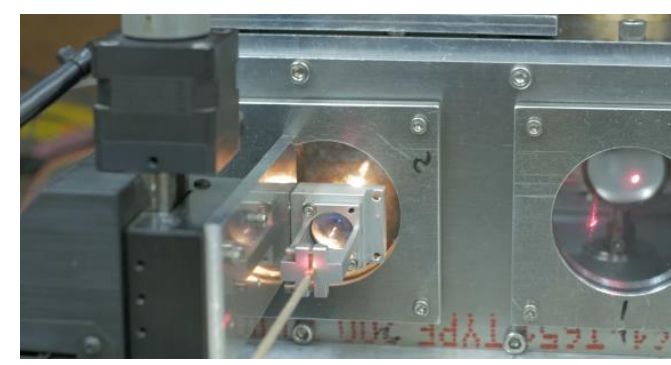

Figure 5. Vacuum chamber to simulate space conditions. High power IR laser on left. Torsion balance readout laser right. reflect off the mirror attached to the torsion balance and into the PSD detector (Figure 5). A vacuum gauge is used at the end of the chamber near the sample to measure the chamber pressure during ablation. Several vacuum gauges are attached to the turbo pump as well. The turbo pump gets to much lower pressure than the chamber due to differential pumping when the target is strongly ablating. This limits our vacuum system low pressure limit in the chamber during the ablation process. This is one of the issues to be rectified in the future. 


\subsection{NOISE AND DAMPENING}

The torsion balance is suspended from a long torsion fiber that allows for fine measurements in the micro Newton range. We estimate the noise of our measurement system by taking data with no applied power from the ablation laser. Vibrations are induced in the fiber system from the environment, including our vacuum pump system. We measure 2-3 $\mu \mathrm{m}$ of noise at the detector. A copper disk is attached to the bottom of the torsion balance that sits a few millimeters away from
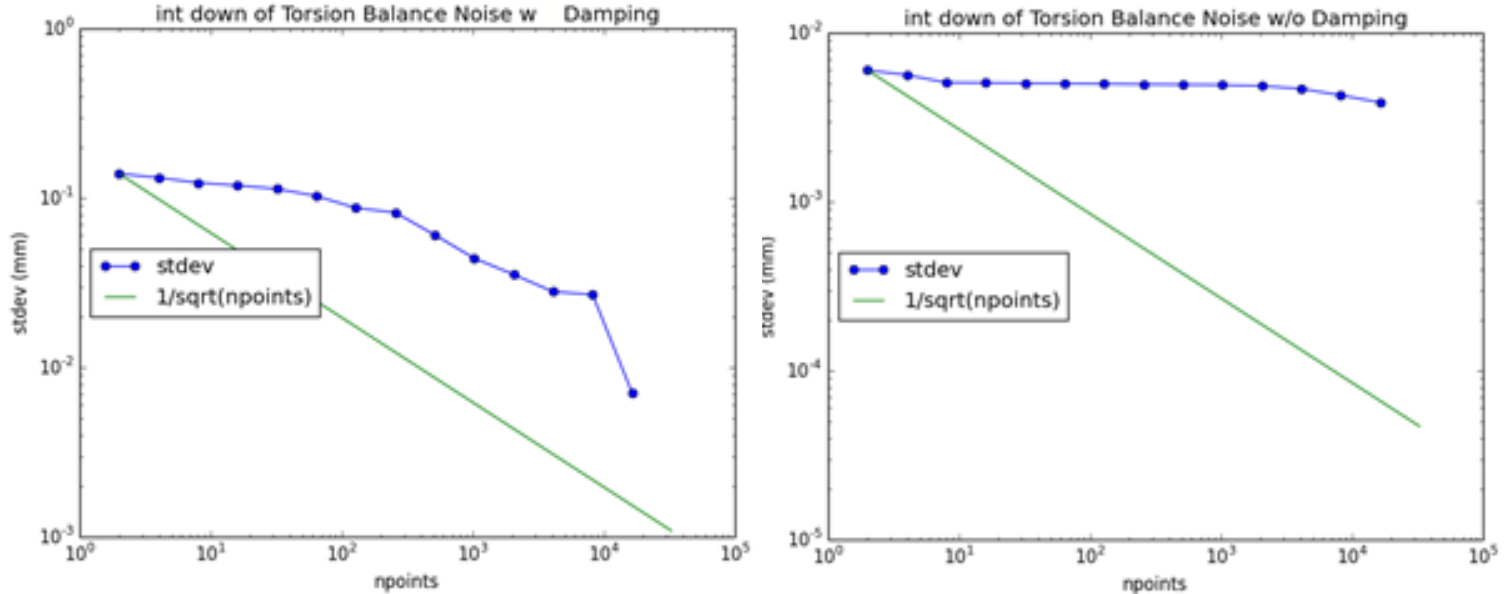

Figure 6. Integration down plot to determine laser ablation data duration. Left includes eddy current dampener to reduce data duration of laser ablation while right doesn't include dampening.

a high field rare earth magnet at the bottom of the chamber. This damps the natural oscillations via eddy-current dissipation. An integration down plot was performed with and without the eddy current dampener to determine the dampening coefficient (Figure 6).

\subsection{Calibration}

In order to measure the ablation thrust we need to determine the torsion constant of the fiber. This is done both theoretically based on the fiber material properties (SS 304 in our case) and then measuring the torsion constant directly to cross check. We have done both and they agree within a 10 percent.

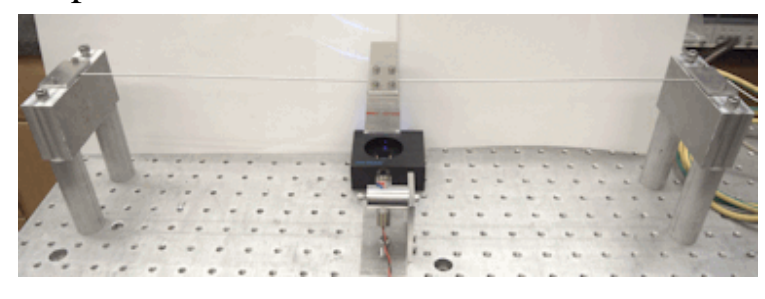

Figure 7. Torsion fiber constant experimental setup. Use known weights on aluminum strip to torque the fiber and use a laser and a mirror to track the distanced moved on the detector.
To measure the torsion constant of our experiment fiber, we devised the system shown in Figure 7. We clamp the torsion fiber at both ends and attached a strip to the middle of the fiber. We apply a torque to the system by placing known masses on the strip and using the measurement laser, a mirror attached to the bottom of the aluminum strip, and the PSD detector. We measure a torsion constant of $0.123 \mathrm{Nm} / \mathrm{rad}$ consistent with our calculations.

\subsection{EXPERIMENT SAMPLE}

We have tried many sample materials. We report the data for porous basalt. Basalt is an aphanitic igneous rock with roughly $20 \%$ quartz, $10 \%$ feldspathoid, and $65 \%$ feldspar in the form of plagioclase. This material was chosen because of the similar composite structure it shares with known asteroid material. It has an average density of $3.0 \mathrm{~g} / \mathrm{cm}^{3}$ depending on sample porosity Due to the rough and porous' nature of our basalt sample, it is difficult to keep the focal length of the laser on the surface of the sample. (Figure 8) 

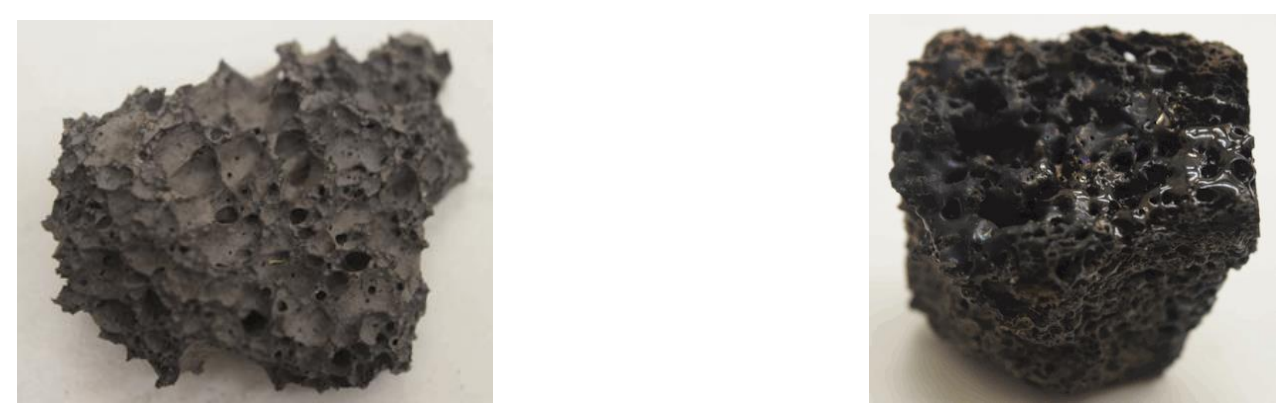

Figure 8. Porous basalt sample that has not undergone any ablation (Left). Sample of basalt from lab testing that has undergone ablation (Right). Both samples come from the same basalt rock.

We have measured the thrust from ambient pressure down to 1 mTorr (approx 6 orders of magnitude). When ablating at near ambient pressure we see very different effects of laser power on the sample when compared to mTorr range pressure.
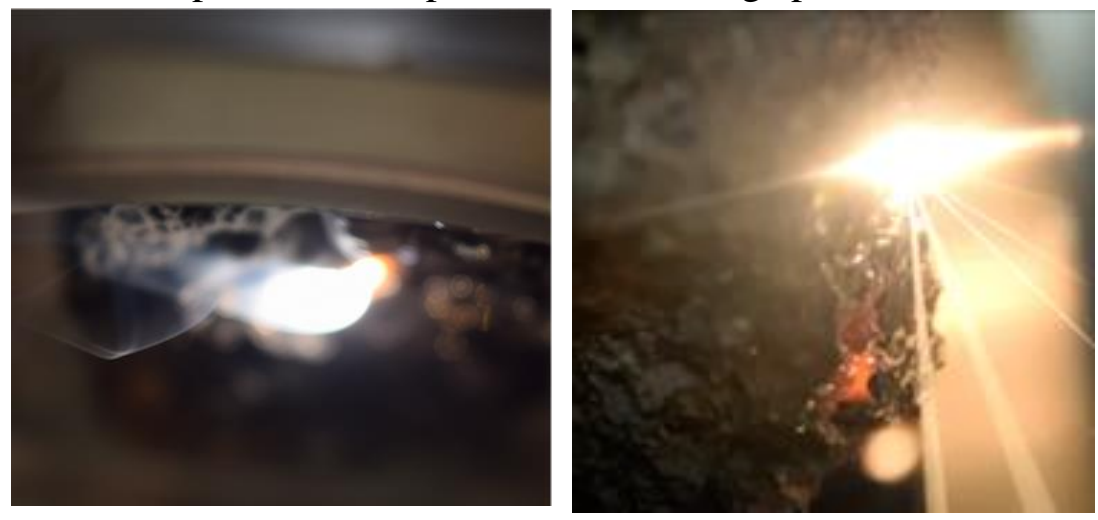

Figure 9. Right photo was taken during a low pressure ablation test and shows mass ejecta and plume. Left photo was taken during an ablation test at ambient pressure and shows the sample smoking and melting.

\section{LASER Power}

The laser is a 19 element fiber coupled system. The fiber and a focusing lens is placed outside of the vacuum on the XYZ stage in order to keep the focus on the surface of the sample. The laser beam has to go through multiple mediums and loses power in each. In particular itgoes through the quartz vacuum window, and quartz blast shield (coated with ejected mass with time) and the ejecta itself as well as the surface melt (sublimation layer). Figure 12 shows the damage to the internal quartz ejecta blast shield. To determine the laser optical power vs. current, a thermocouple laser power meter measures the temperature of the surface the laser hits and is calibrated so a given delta $\mathrm{T}$ corresponds to a given power. The current threshold at which the laser begins to start "lasing" is

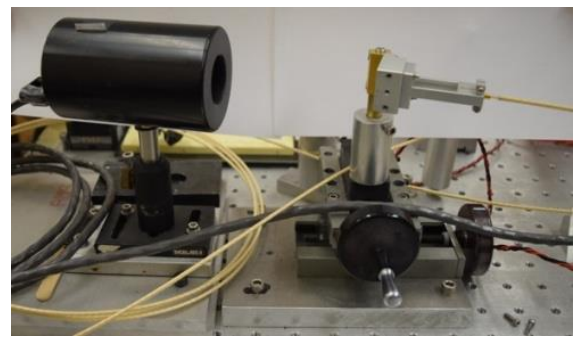

Figure 10. Ablation laser directed at the Power Meter. Power meter was used to determine the slope efficiency of the ablation laser up to 20 amps. 
about 10 amps. Our laser power meter has a maximum input power of 10 watts (Figure 10). The laser is expected to be and is measured to be extremely linear with current. (Figure 11).

The slope efficiency of the laser is 1 watt/amp after the current threshold is achieved. There are several critical issues that impact the final flux on the sample. First the power that arrives at the target has to pass through two uncoated quartz (n $1.46 @ 0.808 \mu \mathrm{m})$ windows which give a minimum reflection per surface (at normal incidence) of about $3.5 \%$ or about $15 \%$ overall loss assuming no blast debris on the inner quartz blast shield window. This is consistent with the power with and without the window that we measured outside the chamber. During ablation, material quickly builds up on the inner quartz shield and reduces the total power depending on the previous exposure. Typically we get a $40 \%$ $70 \%$ loss from this effect. We clean and change the windows frequently (Figure 12). The ejecta comes out at $\sim 1 \mathrm{~km} / \mathrm{s}$ and can severely damage the quartz.

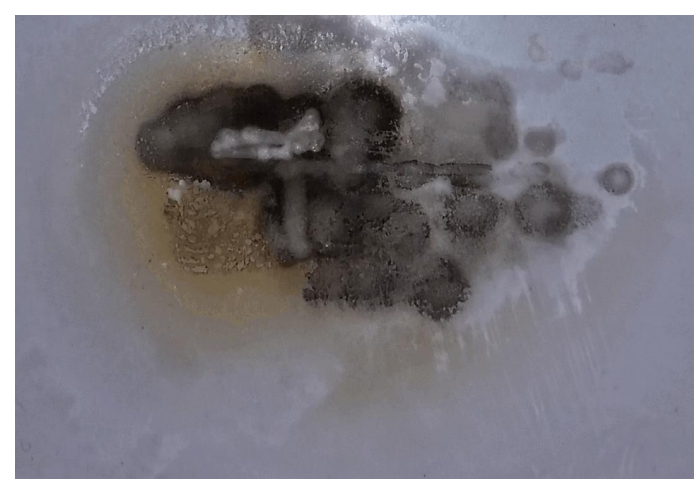

Figure 12. Laboratory experiment quartz blast shield that has undergone multiple ablation measurements. The ring of brown debris shows basalt sample ejecta from recent measurement. The black shows ejecta from the multiple laser ablation measurements embedded in the quartz.

\section{Measurements Results}

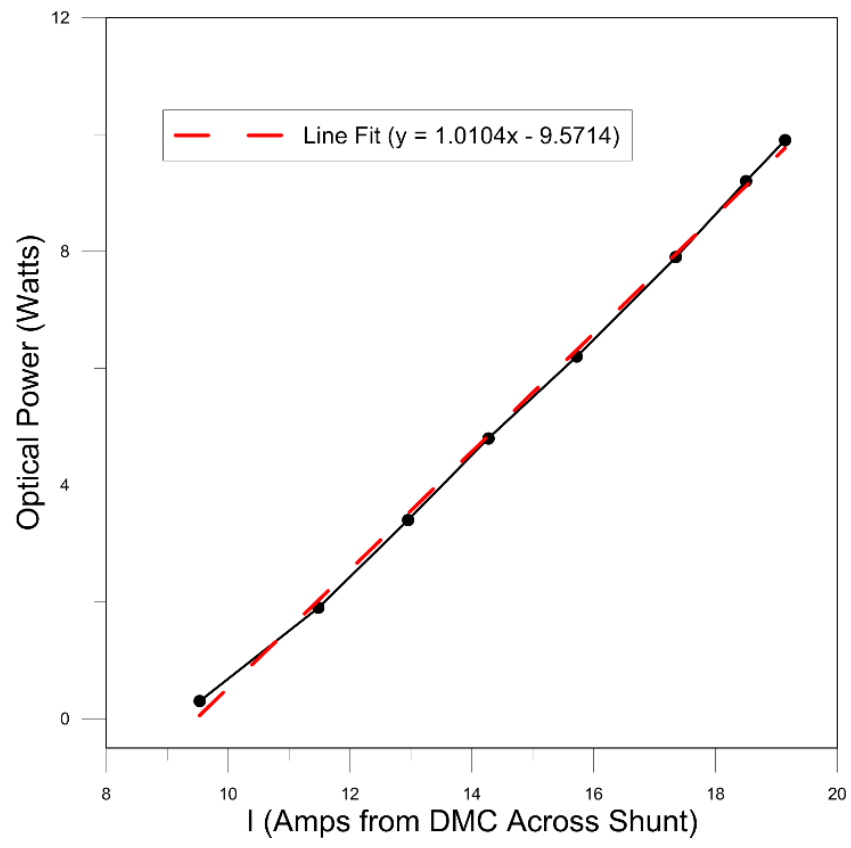

Figure 11. Optical Power Test of the 40W-808NM ablation laser. Test was done with the fiber optic cable and lens directing the laser into the power meter.

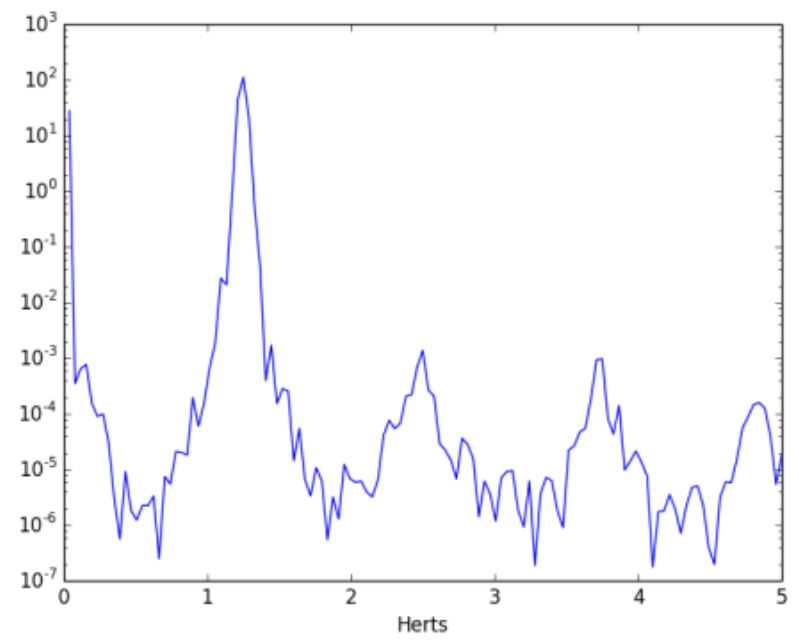

Figure 13. Resonance frequency of torsion balance at $1.1 \mathrm{~Hz}$. Used to determine where to low pass the data.

As mentioned before, the PSD detector outputs a voltage proportional to the displacement of the centroid of the measurement laser spot. We calibrate this voltage to plot displacement on the detector versus time. The natural frequency of the fiber and balance beam was found to be $1.1 \mathrm{~Hz}$ by observing oscillations driven by vibrational noise. (Figure 13) The data was low software pass filtered at $1 \mathrm{~Hz}$ in order to smooth over the resonant oscillations of the torsion fiber and make it easier to see the longer timescale reaction of the beam to the imposed force from laser ablation. 
Our final low pass data plots are used to determine the maximum thrust reached at a given chamber pressure and laser power.

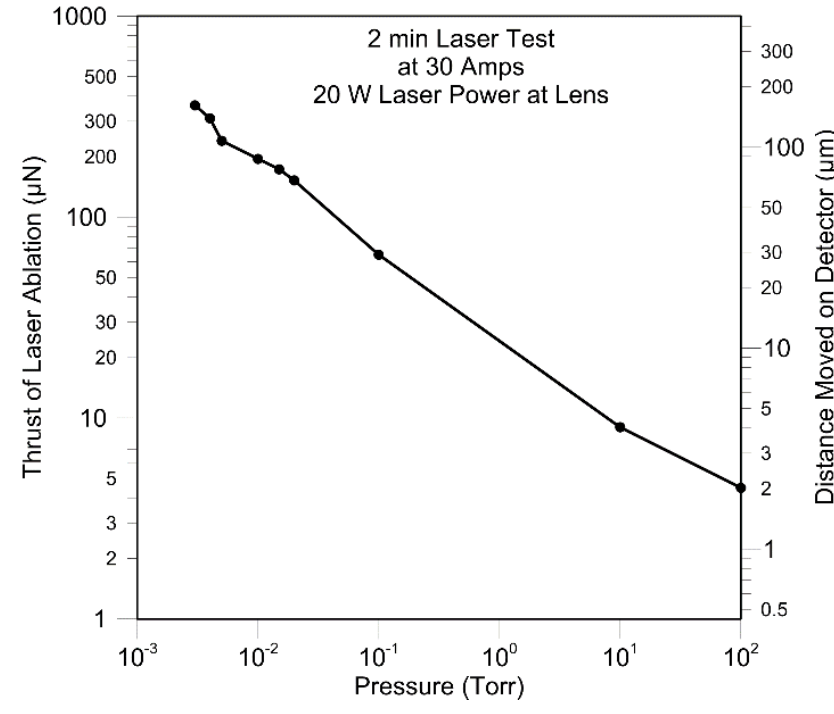

Figure 14. Thrust and Distanced Moved on Detector Vs. Pressure. IR ablation laser power is $20 \mathrm{~W}$ at the lens. Power inside the chamber is $\sim 17 \mathrm{~W}$ and in central spot and absorbed is $\sim 8 \mathrm{~W}$.

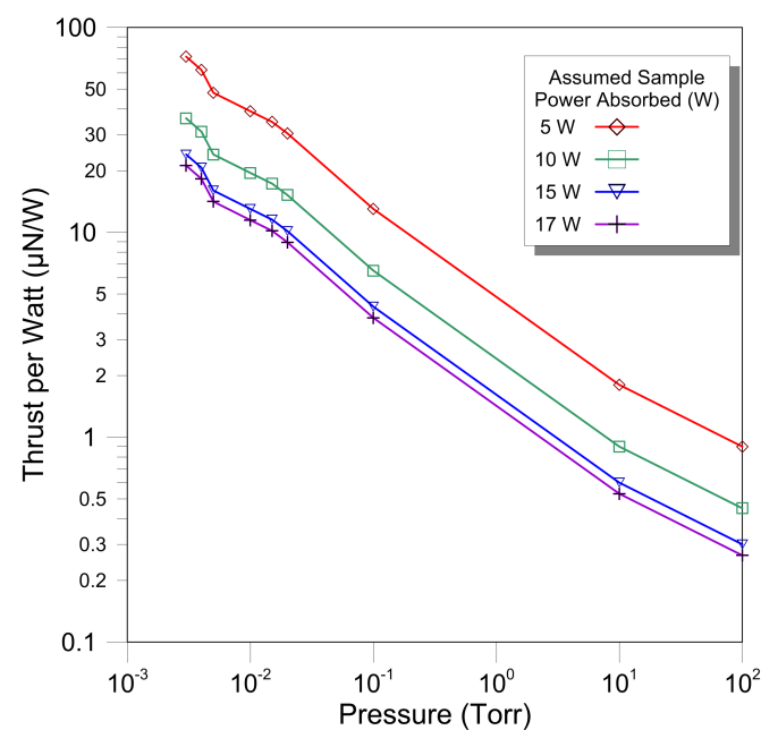

Figure 15. Momentum Coupling Coefficient for various assumed power absorbed by sample. Each line represents a different power absorbed by the sample for $20 \mathrm{~W}$ at outside lens. thus become a lower limit to what will be encountered in a real target. Hence our measurements are extremely conservative compared to what can be achieved in an optimized system. yields about $20 \mathrm{~W}$ of laser power outside the chamber and $17 \mathrm{~W}$ inside the chamber assuming no debris on the inner window. Taking into account various issues including the effective beam and useful (central spot) flux on the target we estimate we are abosrbing about $50 \%$ of this in the critical central spot. This would give about $8 \mathrm{~W}$ absorbed. We measured a thrust of $360 \mu \mathrm{N}$ for our lowest for a total laser power of $20 \mathrm{~W}$ (outside the chamber), $17 \mathrm{~W}$ inside and the assume $8 \mathrm{~W}$ absorbed as discussed above. Using this we calculated a thrust per watt of 360/8 45 $\mu \mathrm{N} / \mathrm{Watt}_{\text {absorbed }}$ (Figure 14).

Several problems arise due to the extreme environment near the sample while ablating. There is a beam focus issue, which for our system (F\# 1) converges rapidly and as the surface is ablated the focus moves into the target. This complicates the estimate of the actual flux delivered to the central spot. Further complications are reflection of the laser from the melted surface and absorption in the ejected plume. The latter appears from our models to be at about the $10 \%$ level. We are investigating the surface reflection from the interaction region. An additional complication is the mode structure of the beam at the spot due to the non-Gaussian nature of the beam. Due to not being able to pump the chamber fast enough to remove the ejecta, we struggle to achieve a hard vacuum which is shown clearly in Figure 15. Even greater thrust appears possible at lower pressures. Since the mass ejection rate and hence the thrust is a very sensitive function of the flux, all these effects become important in comparing our measured results to our models. Our measurements of thrust
We typically run the IR laser at 30 amps which pressure to date (which is still not low enough) 


\subsection{Mass Ejection Photos ANd Video}

Below are a variety of images taken while ablating at low pressure. These pictures show the complex interaction region at the target surface of the basalt target while ablating. We get a wide range of detail about the ablation process that is occurring. There is bubbling, mass ejecta, sparks, and plume clouds. An HD Video containing multiple clips is here: http://www.deepspace.ucsb.edu/projects/directed-energy-planetary-defense
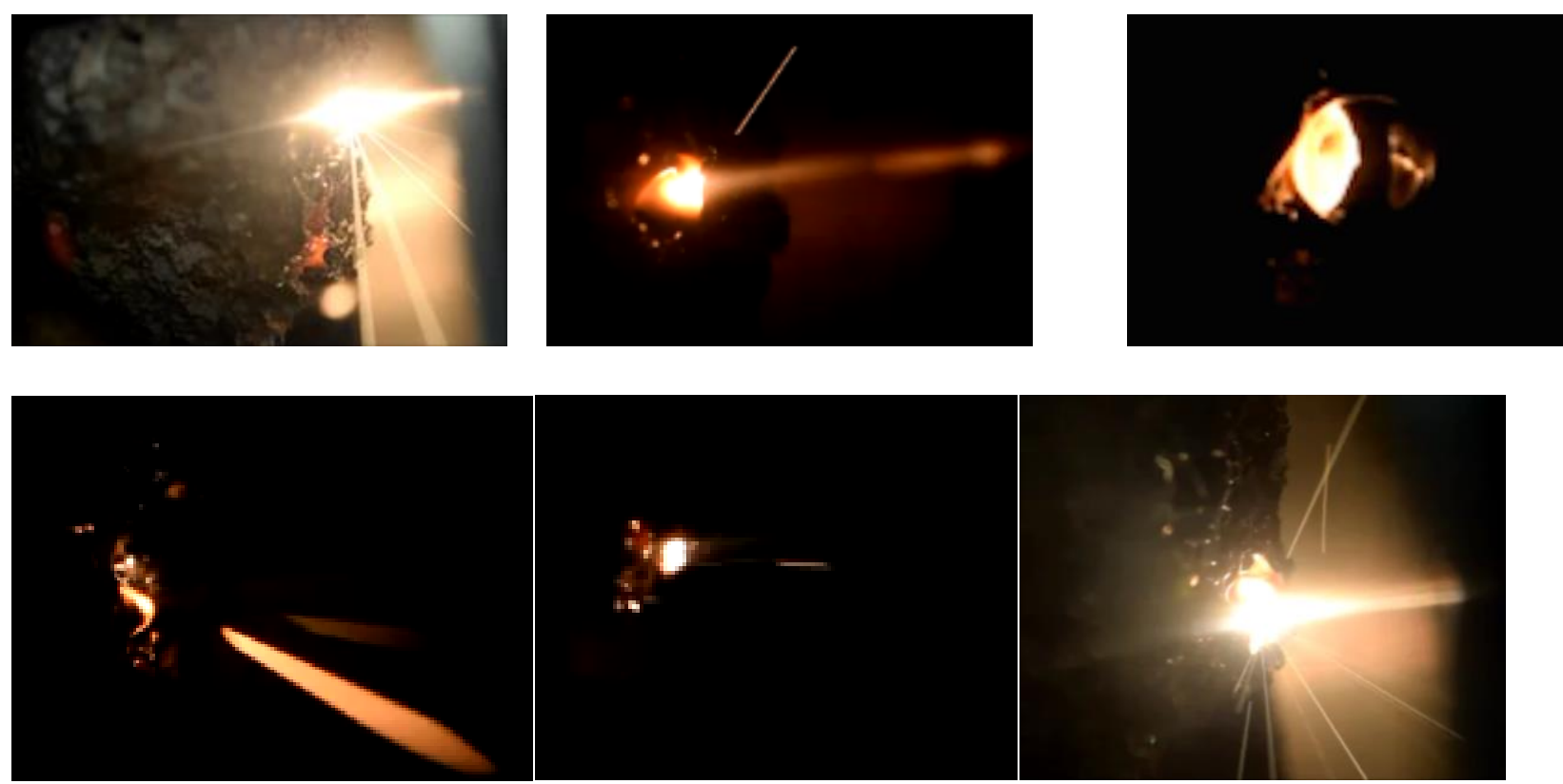

Figure 16. Various pictures of mass ejecta, sparks, plume, and bubbles due to laser ablation. These pictures show the environment at the sample while laser ablation is occurring. See online video.

\section{CONCLUSIONS AND FUTURE Works}

We have shown that directed energy mitigation of asteroids is a feasible method of deflecting threats and that laboratory measurements of this approach are reasonably consistent with our analytic and numerical simulations. Much more work is needed to explore optimization of the system. In the future we will increase the chamber size, increase the laser power, optimize the optics to increase the central flux and further automate the system. We will also have a real time servo controlled focusing system to optimize the thrust. There are numerous system diagnostics that need to be implemented including ejecta and beam profiling measurements.

\section{ACKNOWLEDGEMENTS}

We gratefully acknowledge funding from the NASA California Space Grant NASA NNX10AT93H in support of this research.

\section{REFERENCES}

[1] Lubin, P., Hughes, G.B., Bible, J., Bublitz, J., Arriola, J., Motta, C., Suen, J., Johansson, I., Riley, J., Sarvian, N., Clayton-Warwick, D., Wu, J., Milich, A., Oleson, M., Pryor, M., Krogen, P., Kangas, M., and O’Neill, H. “Toward Directed Energy Planetary Defense,” Optical Engineering, Vol. 53, No. 2, pp 025103-1 to 025103-18 (2014).

[2] Kosmo, K., Pryor, M., Lubin, P., Hughes, G.B., O’Neill, H., Meinhold, P., Suen, J., C., Riley, J., Griswold, J., Cook, B.V., Johansson, I.E., Zhang, Q., Walsh, K., Melis, C., Kangas, M., Bible, J., Motta, Brashears, T., Mathew, S. and Bollag, J. "DE-STARLITE - a practical planetary defense mission," Nanophotonics and Macrophotonics for Space Environments VIII, edited by Edward W. Taylor, David A. Cardimona, Proc. of SPIE Vol. 9226 (Aug, 2014). 
[3] Hughes, G.B., Lubin, P., Griswold, J., Bozinni, D., O’Neill, H., Meinhold, P., Suen, J., Bible, J., Riley, J., Johansson, I.E., Pryor, M. and Kangas, M. "Optical modeling for a laser phased-array directed energy system (Invited Paper)," Nanophotonics and Macrophotonics for Space Environments VIII, edited by Edward W. Taylor, David A. Cardimona, Proc. of SPIE Vol. 9226 (Aug, 2014).

[4] Johansson, I.E., Tsareva, T., Griswold, J., Lubin, P., Hughes, G.B., O’Neill, H., Meinhold, P., Suen, J., Zhang, Q., J., Riley, J. Walsh, K., Brashears, T., Bollag, J., Mathew, S. and Bible, J. "Effects of asteroid rotation on directed energy deflection," Nanophotonics and Macrophotonics for Space Environments VIII, edited by Edward W. Taylor, David A. Cardimona, Proc. of SPIE Vol. 9226 (Aug, 2014).

[5] Lubin, P., Hughes, G.B., Kosmo, K., Johansson, I.E., Griswold, J., Pryor, M., O’Neill, H., Meinhold, P., Suen, J., Riley, J., Zhang, Q., Walsh, K., Melis, C., Kangas, M., Motta, C., and Brashears, T., "Directed Energy Missions for Planetary Defense," in press, Advances in Space Research - Special Edition, Elsevier 2015.

[6] Lubin, P. and Hughes, G.B., "Directed Energy Planetary Defense," invited chapter in "Handbook of Planetary Defense", Springer Verlag, in press 2015.

[7] Popova, O.P., Jenniskens, P., Emel'yanenko, V., et al. "Chelyabinsk Airburst, Damage Assessment, Meteorite Recovery, and Characterization," Science: Vol. 342 no. 6162 pp. 1069-1073, 29 November 2013.

[8] Glasstone, S., and Dolan, P. The Effects of Nuclear Weapons, Third Edition, Washington: Department of Defense, 1977 , ch. 2.

[9] Morrison, D, Harris, A.W., Sommer, G., Chapman, C.R. and Carusi, A. "Dealing with the impact hazard." Asteroids III (ed. W. Bottke et al., Univ. Ariz. Press) (2002): 739-754.

[10] Gibbings, M. A., Hopkins, J. M., Burns, D., \& Vasile, M. (2011). “On Testing Laser Ablation Processes for Asteroid Deflection,” 2011 IAA Planetary Defense Conference, Bucharest, Romania.

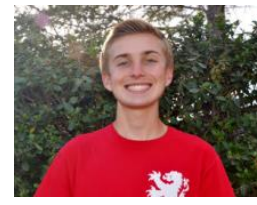

Travis Brashears is currently working in the Physics Department at the University of California at Santa Barbara in the Experimental Cosmology Group. His primary research is on the possibility of deflecting earthbound asteroids via laser ablation, as well as the application of the same laser technology to propel spacecraft for interstellar exploration.

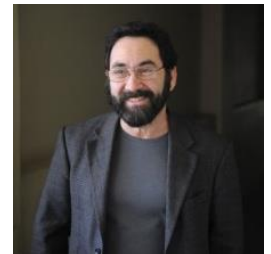

Philip Lubin is a professor of Physics at the University of California, Santa Barbara. He received his $\mathrm{Ph}$.D. in Physics from UC Berkeley. His primary work is in studies of the early universe and he is co-recipient of the Gruber Prize for Cosmology in 2006. He has more than 3 decades of experience in designing, building and deploying far IR and $\mathrm{mm}$ wave systems for ground, airborne and orbital applications. 